\title{
Layered Manufacture of Multi-Level of Detail Models
}

\author{
A. Fischer, S. Azernikov \\ Laboratory for Computer Graphics and CAD, \\ Dept. of Mechanical Engineering \\ Technion, Haifa, Israel \\ tel: 972-4-8293260, fax: 972-4-8324533, \\ e-mail: meranath@tx.technion.ac.il
}

\begin{abstract}
For layered manufacturing, a 3D CAD model has to be converted from a solid representation to a $2 \mathrm{D}$ layered cross-section. The process usually involves face triangulation or meshing of a sculptured object and then extraction of layers from the meshed models. In current systems, the resulting model suffers from topological problems such as degenerate facets, undesired holes or flipped normals, resulting in incomplete cross-sections that cannot be manufactured as layers. Therefore, time consuming processes for facet repair were added to these CAD systems. This paper proposes a new method for extracting the layers and overcoming the topological problems. In this method, a mesh is reconstructed from the 3D model and represented in multi-level hierarchical structure. The proposed method utilizes the hierarchical model by extracting the layers in real time based on the incremental marching cube algorithm. Moreover, during construction, the method checks the topology and creates a valid cross-section that can be manufactured as layers. The method is applied to multi-level models so that a layered part can be constructed at any desired Level Of Detail (LOD), with rough or fine layers according to the application requirements. Several examples for layered manufacturing of multi-level models will be demonstrated.
\end{abstract}

Keywords

Rapid prototyping, layered manufacturing, multi-level of detail models 


\section{INTRODUCTION}

Layered manufacturing is the prevailing technology in rapid prototyping application. In layered manufacturing, a 3D CAD model has to be converted from a solid representation to a 2D layered cross section. The layer should be well defined with closed boundaries that determine the material areas of the layer. The process usually involves face triangulation or meshing of a sculptured object and then extraction of cross sections from the meshed models (STL format). In current systems, the resulting model suffers from topological problems such as degenerate facets, undesired holes or flipped normals leading to uncompleted cross sections that cannot be manufactured as layers. Therefore, time consuming processes for facet repair were added to these CAD systems. As part of the process the isocurves are extracted and converted to closed boundaries, in order to guarantee valid layer from topological point of view.

In this paper, the multi-level structured will be utilized for the process for layer extraction. The paper proposes a new method for extracting the layers while overcoming topological problems. Using this method, a mesh is reconstructed from the 3D sampled model and represented in multi-level hierarchical structure. The proposed method utilizes the hierarchical model by extracting the layers in real time based on the incremental Marching Cube algorithm. The algorithm can handle the case of neighboring nodes that belong to different levels.

During construction, the proposed method checks the topology of the layer and creates a complete cross section that can be manufactured as a layer. The method is applied to multi-level models so that a layered part can be constructed at any desired Level Of Detail (LOD): Rough or fine layers according to the application requirements. Moreover, a mixed LOD structure can be constructed by integrating areas from different LODs. In the proposed system, a selected area can be marked as a high resolution area and, as a result, a refined adaptive mesh is constructed. The resulting object can be manufactured in rough or fine details according to this area selection. This mixed LOD process makes it possible to produce only interesting areas with fine details, while all the rest can be in the form of a general draft. In this paper, the multi-level model is described in Section 2. In Section 3, the rapid prototyping process based on the multi-level model is described and several examples of layered manufacturing are demonstrated. A summary and conclusions are provided in Section 4.

\section{THE MULTI-LEVEL OF DETAIL MODEL}

Representing an object with hierarchical multi-levels of detail (LOD) structure is essential for design, modeling and analysis. The LOD method consists of two main stages: 1) constructing a hierarchical LOD structure and (2) designing, analyzing and displaying the 3D model at different levels of details. With the new LOD approach, the multi-level model is constructed a priori; then, any level of detail can 
be accessed directly in real time, according to application requirements. Moreover, transition between the levels can be achieved in real time. In addition, the LOD construction satisfies the condition that the geometric and topological behavior is preserved at each level under a given tolerance. That is, even at lower resolution levels, critical details are preserved (Figure 1). For all of these reasons, the multilevel structure, which represents the object at different level of abstraction, is superior to conventional models for CAD/CAM applications.

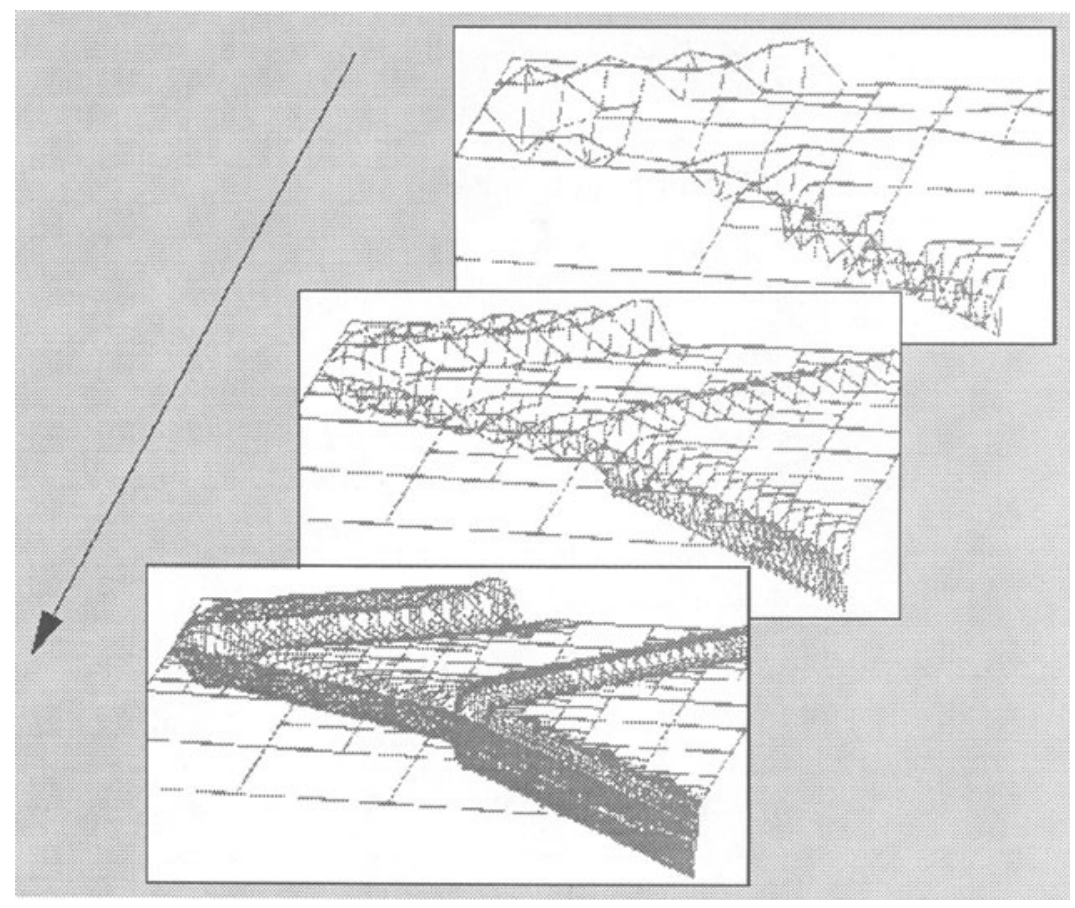

Figure 1: Several level of detail meshes from low to high level. Any level can be extracted according to a given error tolerance.

The two most common multi-level structures are the multiresolution wavelet technique (Lucier, 1992; Stollnitz et al., 1995) used for continuous representation, and the LOD technique used for discrete representation (Finkelstein et al., 1996; Lindstrom et al., 1996). The multiresolution technique is usually used for objects represented by sculptured surfaces that are naturally designated by a high degree polynomial, while the discrete representation is used for 3D mesh representation. This paper focuses on 3D mesh representation for both discrete and continuous representations. BR is represented directly as a mesh, while a freeform surface is represented by its control polygon mesh. 
The proposed system for displaying meshes using LOD technique is described as follows (Figure 2):

\section{LOD Display Algorithm}

Diplaying quadtree nodes according to accumulating error norm

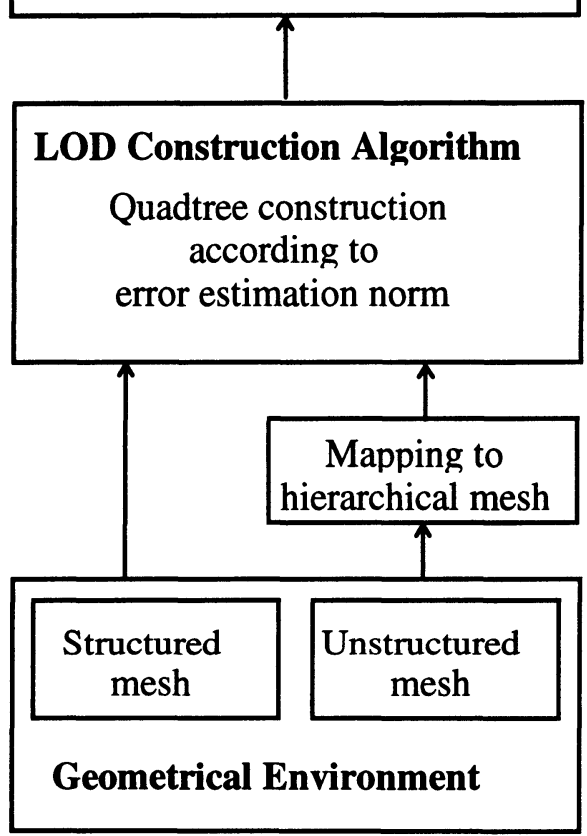

Figure 2: The LOD approach scheme.

(a) The geometrical representation in the proposed system can be a uniform mesh or an unstructured mesh. The system can also handle analytic surfaces which have been made discrete through uniform or adaptive hierarchical or unstructured meshes.

(b) In the system, the uniform meshes are represented in hierarchical Quadtree data structure. This structure is constructed according to error criteria explained in Section 2. The construction is implemented in a TD strategy. Then, the resulting nodes represent a square region where its vertices are points on the mesh with $(\mathrm{x}, \mathrm{y})$ geometric value (position) and $\mathrm{z}$ value represented as pseudo color. For the unstructured mesh case, a pre-process is applied to distribute the nodes in an adaptive hierarchical structure according to the density of the mesh nodes. 
(c) In the display stage, the approximated mesh can be displayed at several levels of resolution according to the LOD error criteria.

\subsection{The Construction Algorithm}

The construction algorithm can be applied on structured or unstructured 3D meshes. The 3D mesh is represented by 3D structure: a Quadtree is built representing the $x-y$ values while a pseudo-color in the nodes represents the $z$ value using Top Down (TD) strategy (Hugues, 1996), fewer nodes are processed compared to Bottom Up (BU) strategy. Moreover, with TD strategy, preservation of the shape at critical high-frequency locations is guaranteed by using geometry (curvature) and/or solution (gradient of values) behavior as criteria. For each node (region) qk, several points are sampled uniformly. An error is evaluated according to the error estimate indicator:

$$
E\left(q_{k}\right)=\left|\sum_{i=1}^{N} \sum_{j=1}^{N}\left(\Delta v_{x}(i, j)-\overline{\Delta v_{x}}\right)^{p}\right|^{1 / p}+\left|\sum_{i=1}^{N} \sum_{j=1}^{N}\left(\Delta v_{y}(i, j)-\overline{\Delta v_{y}}\right)^{p}\right|^{1 / p}
$$

where $\mathrm{NxN}$ is the number of sample points in region $\mathrm{qk}, \Delta \mathrm{v}_{\mathrm{x}}, \Delta \mathrm{v}_{\mathrm{y}}$ are the values of the color differences, and $\Delta \mathbf{v}_{\mathbf{x}}, \Delta \mathrm{v}_{\mathrm{y}}$ the values of the average color differences in the $\mathrm{x}$ and $\mathrm{y}$ directions, respectively. When the process terminates, the error of each node is re-estimated in a Bottom Up strategy with respect to the finest level according to the accumulated error indicator:

$$
E\left(q_{k}\right)=\frac{\left[\int_{\Omega_{k}}\left|\left(M_{n}-M_{0}\right)^{P}\right| d \Omega_{k}\right]^{1 / p}}{\left(\Delta x_{k} \Delta y_{k}\right)^{\alpha}}
$$

where $\mathrm{Mn}-\mathrm{M} 0$ is the difference between the $\mathrm{z}$ values of the meshes $\mathrm{Mn}$ and $\mathrm{M} 0$. Thus, the accumulated error is attached to each node and serves as the base for the LOD display algorithm.

\subsection{Level of Detail Extraction}

The LOD extraction algorithm that extracts one level from the multilevel model can work according to one of the following optimal functions: (1) Given a number of elements, find the mesh with the minimal error. (2) Given an error tolerance, find the mesh with a minimal number of elements. The first function is implemented for time dependence simulation, where the rate of the frames should be constant; in the second option, it is used for static meshes that are displayed or 
analyzed progressively. Currently we deal with static meshes and, therefore, a reduced mesh is extracted with a minimal number of elements according to a given display error tolerance (Fig. 4). This tolerance defines the level of detail that will be extracted according to the application requirements. The extraction algorithm checks the nodes recursively using TD strategy.

\subsection{Discontinuity Effect}

In this work, it is assumed that the original geometrical and physical model is smooth with at least $\mathrm{C}_{0}$ continuity. This continuity is therefore constrained in the approximated mesh model. However, hierarchical quadtree-based meshes are not matching models (De Floriani et al., 1996) and are not capable of naturally preserving $\mathrm{C}_{0}$ geometric continuity along element edges. Therefore, special treatment is required to connect the common edge between two elements of different sizes in order to eliminate discontinuities. Several techniques have been suggested in the literature for coping with this problem. Von Herzen and Bar (1987) were the first to propose a method which is common today, known as "restricted quadtree." According to this method, two neighboring elements in the tree are allowed to differ by at most one level; then, triangulation is performed at the element level in accordance with a case table. The constrained meshes used by this method are very rigid. This inflexibility constitutes a major disadvantage, especially considering that structured meshes are already much less adaptable than unstructured meshes. This article proposes a quadtree mesh that allows a difference of more than one level between neighboring elements while still maintaining $\mathrm{C}_{0}$ continuity along element edges. Thus, the mesh can adapt itself more efficiently and can describe the geometry more compactly. The proposed connecting process consists of two main stages:

1. For each element:

* All the neighboring elements are searched and identified using the NeighborFinding Algorithm (NFA) (Samet, 1990). This algorithm makes it possible to find all those neighbors whose level is higher or equal to that of the element being checked.

* To find those neighbors whose level is lower than the checked element, a table was defined outlining the lower level neighbors of each of the four edges. For a given side (North, South, West, East) of the current element, GET_SMALLER_NEIGHBORS, a recursive function, returns a list of all the lower-level neighbors (see Figure 3). 


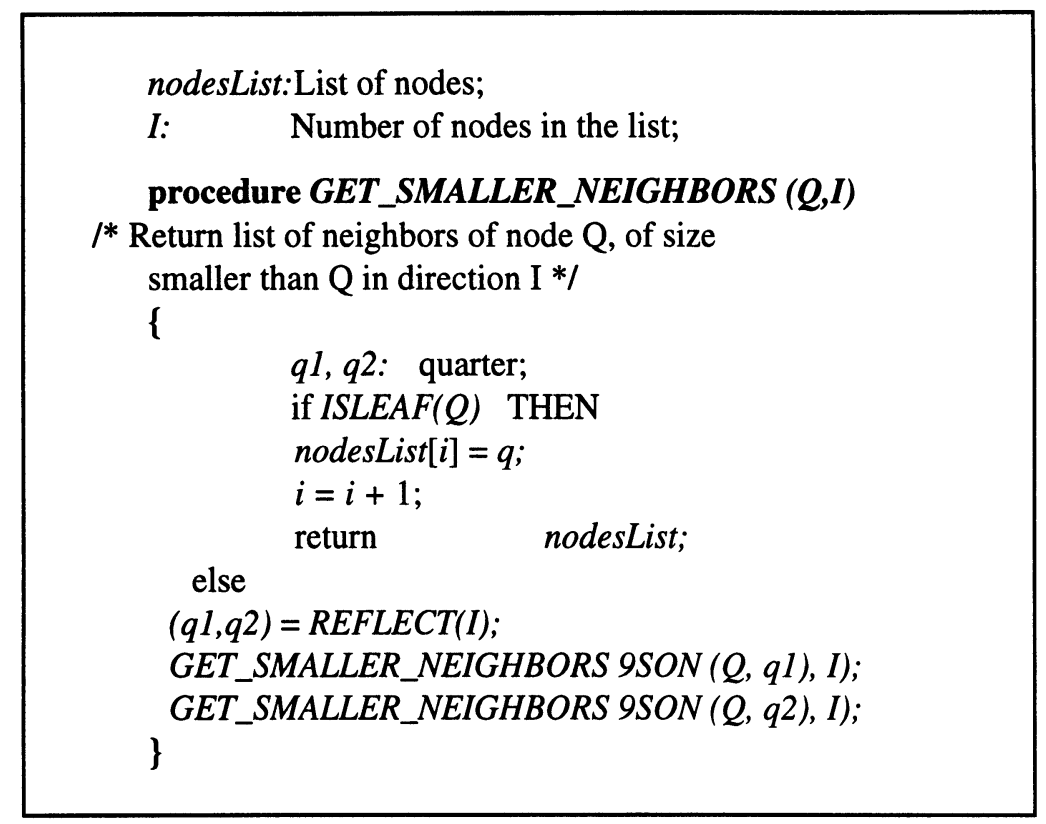

Figure 3: NFA chart Reflect(I).

2. Then, standard Open GL functions are employed for meshing the non-planar element and interpolating between its vertices.

The search algorithm based on the proposed table has a time complexity dependence $\mathrm{O}\left(\mathrm{N}^{*} \log (\mathrm{n})\right)$, where $\mathrm{N}$ is the number of neighbors and $n$ is the number of nodes in the tree.

\section{RAPID PROTOTYPING}

\subsection{Extraction of Manufactured Layers}

For the rapid prototyping application, layers are extracted from the 3D multi-level mesh. The 3D mesh can represents a sculptured surface or discrete data. The layers are extracted from the multi-level structure where the topological structure is determined according to the following stages:

1. Discontinuity is checked (Section 2.3) and, if needed, elements are connected. For rapid prototyping models, $\mathrm{C}_{0}$ continuity is essential for guaranteeing valid layers.

2. Iso-parametric curves are extracted. The iso-parameter is determined according to the z-value. An algorithm for iso-parametric curves based on the Marching Cube method (Finkelstein et al., 1996) was developed and implemented. The 
algorithm works in real time and can handle the case of neighboring nodes that belong to different levels.

3. The orientation of the segmented curves is determined sequentially and in one direction.

4. The iso-curves are used as a boundary of the layers. Closed contours (boundaries) are created. Segmented curves are closed where discontinuities are revealed (Fig. 5a).

5. For each layer, the material side of the contour is determined. To determine the material side of each layer, inner points should be checked. Since all the contours are oriented consistently, it is relatively easy to find the inner side of the contour (Fig. 5b).

For low level meshes, relative to high level of meshes, the number of elements is significantly smaller and the computation time is therefore faster. However, the boundary resolution of the layers that were extracted from the high level structure are smoother than those at the low level. Figures 4 and 5 demonstrate a mouse surface at several LOD levels with the extracted layers for the rapid prototyping process.

\subsection{Layers with Adaptive Variable Thickness}

In conventional layered manufacturing the thickness of the layers is constant and uniform. The thickness is determined according to a given tolerance parameter (Lee et al., 1998; Kullkarni and Dutta, 1996). However, creating all the layers with similar thickness according to the highest resolution is redundant, and an adaptive process is desired (Kullkarni and Dutta, 1996). In the proposed conventional algorithm, the cross-sections are created uniformly according to constant and uniform $\mathrm{z}$ values. The iso-curves of each layer are computed by the marching cube algorithm. This algorithm does not consider the adaptive element size of the mesh.

However, such extension is natural. The 3D geometrical shape is already represented in adaptive 3D mesh structure where the size of the elements is according to shape criteria; the mesh is refined adaptively in the areas where the curvature is high. Therefore, this adaptive mesh can be used as a base for the process of creating the adaptive layers. In the adaptive approach, the Marching Cube algorithm is extended. During 'marching' between the elements, the maximum $\mathrm{z}$-value of the elements is computed. This max value dictates the $\mathrm{z}$ value of the next layer. For example, if the geometry is relatively flat, the elements are thick and the number of layers is small. In contrast, in a case where the shape is highly curved, the elements are relatively thin and the number of layers is higher than in the case of flat geometry. The advantage of this method is that the extension is straightforward. The number of layers is reduced significantly while the error is preserved. In addition, in the multi-level representation, for each level, the number of layers and their boundary resolution changes proportionally to the detail resolution. 


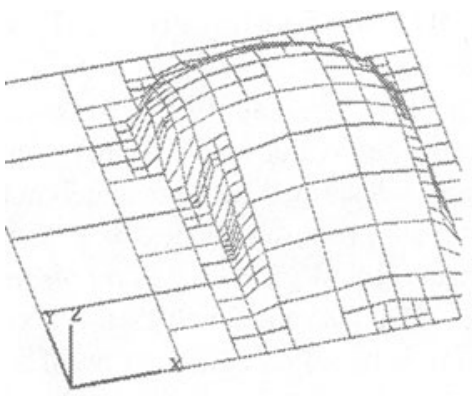

(a)

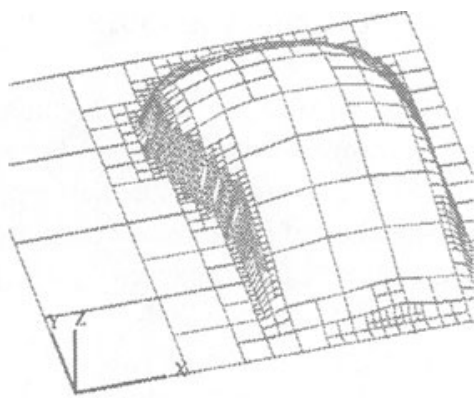

(b)

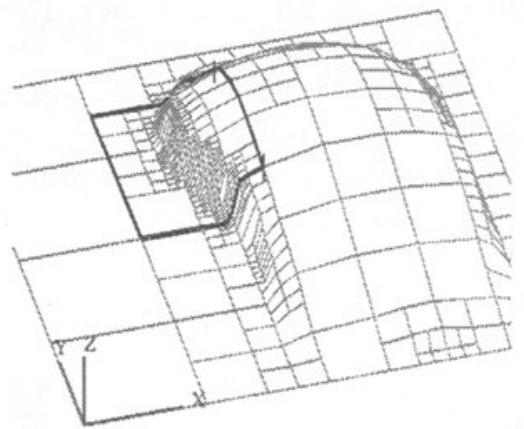

(c)

Figure 4: A mouse object (a) Low resolution; (b) high resolution; (c) mixed resolution focusing on a particular area with high resolution.

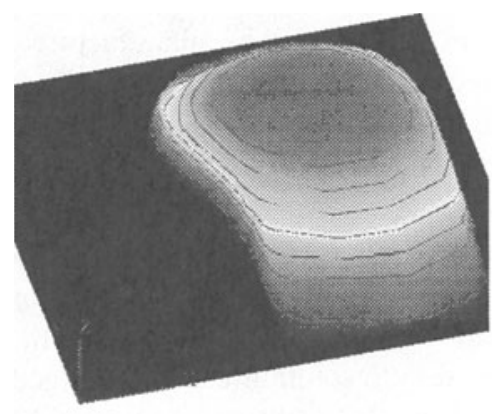

(a)

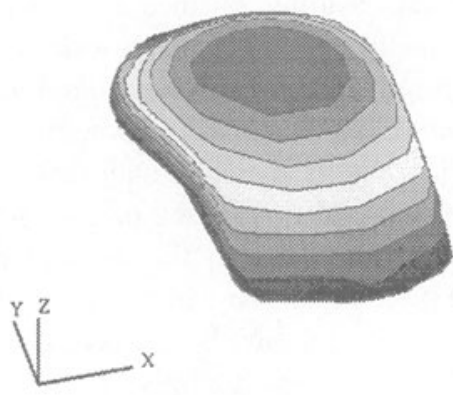

(b)

Figure 5: A mouse model: (a) a mouse model with the extracted iso-curves; (b) extracted layers for rapid prototyping. 


\subsection{Generalizing the LOD technique to 3D closed volumetric objects}

So far, the construction and extraction algorithms have been applied to 3D meshes which represent a surface model. For such objects, the z-values were represented in a pseudo-color solution, and the (x,y) values were described on planar geometry. For 3D meshes of 3D closed volumetric objects, the proposed process is based on the concept of texture mapping. The 3D complex closed geometry is represented as a parametric surface $\mathrm{X}(\mathrm{u}, \mathrm{v}), \mathrm{Y}(\mathrm{u}, \mathrm{v}), \mathrm{Z}(\mathrm{u}, \mathrm{v})$. The curvature values are texture mapped on the 3D geometry. Mesh reconstruction is based on construction of a $3 \mathrm{D}$ pure geometric mesh for the 3D object according to curvature criteria. The 3D mesh is refined according to these curvature values. This stage uses the same algorithm as that for the 3D surface model.

In analysis, a solution might be added as a texture and mapped to the geometry. For a case where the geometry mesh is changed according to resolution requirements, only the geometry mesh should be reconstructed. For a case where the solution resolution is changed and the geometry is fixed, only the texture mapping is reconstructed. This method can be applied to the adaptive slicing method where the variable thickness is mapped as a texture to the geometry and can be refined accordingly.

\section{SUMMARY AND CONCLUSIONS}

This paper proposes a new method in the area of rapid prototyping for extracting the manufactured layers from LOD structure. The method overcomes conventional topological problems, such as such as degenerate facets, undesired holes or flipped normals leading to uncompleted cross sections that cannot be manufactured as layers. In this method, a mesh is reconstructed from the 3D surface model and represented in multi-level hierarchical structure. Currently, the system is being extended to 3D closed volumetric objects.

The proposed method utilizes the hierarchical LOD model by extracting the layers in real time based on the incremental Marching Cube algorithm. Moreover, during construction, the method checks the topology and material, creating a complete layer that can be manufactured. With the multi-level representation, for each level, the number of layers and their boundary resolution can be changed in real time, proportionally to the detail resolution. In addition, a mixed LOD structure can be constructed by integrating areas from different LODs.

\section{ACKNOWLEDGMENTS}

This research has been supported in part by the Israeli Ministry of Science and the Korean Ministry of Science and Technology as part of the Bi-National IsraelKorea Project No. 6121-1-98. 


\section{REFERENCES}

Certain, A., Popovic, J., DeRose, T., Duchamp, T., Salesin, D., and Stuetzle, W. (1996) Interactive Multiresolution Surface Viewing. ACM Computer Graphics, SIGGRAPH, 91-98.

De Floriani, L., Marzano, P., and Puupo, E. (1996) Multiresolution Models for Topographic Surface Description. Int. J. of Visual Computer, 12/7, 317-345.

Eck, M., DeRose, T., Duchamp, T., Hoppe, H., Lounsbery, M., and Stuetzle, W. (1995) Multiresolution of Arbitrary Meshes. ACM Computer Graphics, SIGGRAPH, 173-180.

Finkelstein, A., Jacobs, C.E., and Salesin, D.H. (1996) Multiresolution Video. ACM Computer Graphics, SIGGRAPH 1996, 281-290.

Hugues, H. (1996) Progressive Meshes. ACM Computer Graphics, SIGGRAPH, 99-108.

Krause, F. -L. and Luddemann, J. (1996) Virtual Clay Modelling. Product Modeling for Computer Integrated Design and Manufacture, Fifth IFIP WG5.2 Workshop on Geometric Modeling in CAD, Virginia, USA, May 1996, Chapman \& Hall, 162-175.

Kullkarni, P. and Dutta, D. (1996) An Accurate Slicing Procedure for Layered Manufacturing, CAD J., 28/9, 683-697.

Lee, K., Hur, J., Ahn, J., and Kim, G. (1998) Geometry Manipulation Tools Applied to Rapid Prototyping Systems. Israel-Korea Conf. on Geometrical Modeling, Tel-Aviv, Feb. 1998, 47-51.

Lindstrom, P., Koller, D., Ribarsky, W., Hodges, L.F., Faust, N., and Turner, G.A. (1996) Real Time Continuous LOD Rendering of Height Fields. ACM Computer Graphics, SIGGRAPH 1996, 109-118.

Lorensen, W.E. and Cline, H.E. (1987) Marching Cubes: A High Resolution 3D Surface Construction Algorithm. ACM Computer Graphics, 21, 163-169.

Lucier, B. (1992) Wavelets and Image Compression. In Lyche, T. and Schumaker, L., eds., Mathematical Methods in Computer Aided Geometric Design, Academic Press, 11, 391-400

Park, S., Song Y.-A., Ha, S., (1998) Comparison between Five Axis Machining and Rapid Tooling for Manufacture of a Fee-Form Shaped Functional Parts. Israel-Korea Conf. on Geometrical Modeling, Tel-Aviv, Feb. 1998, 47-51.

Samet, H. (1990) Applications of Spatial Data Structure. Addison-Wesley, Reading, Massachusetts.

Stollnitz, E.J., DeRose, T.D. and Salesin, D.H. (1995) Wavelets for Computer Graphics, IEEE Computer Graphics and Appl., May 1995, 76-84.

Von Herzen, B. and Bar, A.H. (1987) Accurate Triangulations of Deformed Intersecting Surfaces. Computer Graphics, 21, 103-110. 


\section{BIOGRAPHY}

Anath Fisher is currently (since 1994) a faculty member in the Department of Mechanical Engineering, Technion-Israel Institute of Technology. She received a B.Sc. (1985) in Computer Science and an M.Sc. (1987) and Ph.D. (1992) in Mechanical Engineering from the Technion. From 1992 to 1994, she was a postdoctoral associate in the Injection Molding Program, Dep. of Mechanical Eng., Cornell University, USA. Her main interests are: geometrical modeling for CAD/CAM, physical-based systems, reverse engineering and rapid prototyping.

Sergei Azernikov completed his B.Sc. in Mechanical Engineering at the Technion and is currently a researcher in the Laboratory for Computer Graphics and CAD in the Department of Mechanical Engineering at the Technion. His main research interests are multiresolution models. 